\title{
Cancer can be Cured $@$
}

\author{
Andrew Hague* \\ President of Cell Sonic Limited, Spain
}

*Corresponding author: Andrew Hague, President of Cell Sonic Limited, Spain.

Received Date: April 21, 2020

Published Date: May 22, 2020

\section{Opinion}

More than three years ago, I worked out how to cure cancer with the CellSonic VIPP machine [1]. There are no side effects, drugs are not used or needed, and the cure is quick, maybe immediate. Since then, hundreds more patients in many countries have been cured with different types of cancers.

At first, we understood that the pressure pulse from CellSonic was the main means by which the tumours were killed. That process is certainly involved and applies in many cases. Within a few months and in conversation with Bradford University where they were diagnosing cancer by measuring permittivity [2], we realised that we were also changing the voltage of the potential across the membrane of the cell to lift it from the $-15 \mathrm{MV}$ of cancer to the $-75 \mathrm{MV}$ of a healthy cell. In other words, we were changing the behaviour of the cell and in that sense it was a cure. The replication of mutant cells ceased, and the correct replication of cells continued.

I sit in the middle of a network linked to CellSonic users around the world and get feedback from them. To know that the protocol [3] on cancer works in all continents on a variety of cancers and patients is the evidence I need to be able to claim to have a cure for cancer. There have been no trials. For the first patient [4], we told him we were confident there would be no harm but could not be sure of success. For forty years, similar technology with higher energy had bombarded the kidneys to smash stones and there were no side effects. No cancers were caused, and none were spread. If there had been any bad news, we would all of heard about it. Millions of patients in urology departments in all countries were being safely treated. That allowed us to use CellSonic on cancer patients [5].
Within three days of the first treatment, the patient declared himself cured. Tests in the following weeks confirmed his own assessment. Many more patients said the same [6]. These were patients paying to be treated. Usually they had already been damaged by chemotherapy and radiation, so their immune system was not working or almost useless and they found a CellSonic Clinic in desperation [7]. Thus, apart from the first patient, there have been no experiments; we could predict the outcome.

It has been interesting to observe how oncologists remain committed to deadly poisons as a cure for cancer. They believe they can selectively kill cancer without harming other parts of the body and when the patient suffers, they say that the patient is not responding to treatment as though it is a failure by the patient. Despite never having found a chemical cure for cancer in a hundred years or more, researchers continue to look for a weakness in cancers of all types and hope to find a poison that will attack the weak area and not damage other areas. There is a constant barrage from charities begging for money to pay for more research in laboratories for a better drug and never do they find a cure but still the public, who are not biochemists, have faith in those in whitecoats because they know no better.

That the cure has come from biophysics [8] is lost on most doctors who were never educated in the science [9]. I thought I was the first to find the cure for cancer. Indeed, I was the first to have an easy to use method that is none surgical and completely without side effects. As Dr Steve Haltiwanger [10] said to me, "I should get a Nobel Prize for medicine if I can live long enough to collect it". The more I explore the literature on my predecessors, the more I learned about non-surgical, irreversible electroporation. 
Invasive electroporation was successfully demonstrated by $\mathrm{Dr}$ Bjorn Nordenström over fifty years ago. At the time, he was also the chairman of the Nobel Price committee and commentators said that his own invention was far better than any that his committee was recommending for prizes. Electroporation was never accepted by the pharmaceutical establishment that ruled medicine in America and Nordenström ended up giving his patents to China where they continued to use it successfully but since then it has disappeared. Certainly, inserting needles almost blindly into a tumour and then another needle adjacent to the tumour to pass a current across is difficult and fiddly. The simplicity of a drug injection was always appealing and if the authorities approved the drug, the doctors were exonerated when the patient died.

CellSonic users are becoming more confident. At first, they could not believe what they saw. As time went on, without understanding the science, they accepted that there was a cause and effect; CellSonic VIPP was causing the cancer to stop mutating. They also grudgingly admitted that they never really understood how drugs were supposed to cure but because they were told to use the drugs they obeyed. It is a pathetic attitude that persists throughout a lot of the profession. Credit to the CellSonic doctors [11] who were thinking for themselves and determined to do better for their patients.

\section{Acknowledgement}

None.

\section{Conflict of Interest}

Author declare no conflict of interest.

\section{References}

1. How the cure for cancer was discovered.

2. Cancer's change of direction.

3. Cancer Cure - the protocol and explanation.

4. Professor Hague's Discovery.

5. Review of reports of curing cancer.

6. Prostate cancer, Poland.

7. Inflammatory Breast Cancer.

8. Cancer and Wounds are similar.

9. Soil, the Immune System and Cancer. Questions Must be Asked!

10. The electrical properties of cancer cells.

11. Skycare Cancer Hospital video. 